\title{
Evaluation of outpatient therapeutic programme for management of severe acute malnutrition in three districts of the eastern province, Zambia
}

\author{
Mike Mwanza, Kufre J. Okop* and Thandi Puoane
}

\begin{abstract}
Background: Severe acute malnutrition (SAM) in children under 5 years of age is a major cause of child mortality during hospital admission worldwide, and is attributed to poor case management. The Outpatient Therapeutic Programme (OTP) is an innovation for treating children with SAM with no medical complications as outpatients within their communities.

The aim of the study was to evaluate the improvement in health outcomes and case fatality rate in children aged 6-59 months diagnosed with SAM and admitted in OTP centres, and to document the barriers to a sustainable OTP intervention in the districts of Eastern Zambia.

Methods: A mixed-methods design was used to assess the health outcomes of OTP intervention. Three districts where OTP centres were operational at the time of study were selected. Records of 390 eligible children admitted with SAM between 2008 and 2010 were reviewed. The health outcomes assessed included recovery and case fatality rates, defaulter rate, and weight gain. Information on the barriers to effective implementation of a sustainable OTP intervention in the districts was collected through semi-structured key-informant interviews with stakeholders. Outcome indicators were compared with the baseline data and recommended minimum standards for therapeutic feeding centres.
\end{abstract}

Results: Of the 390 children admitted into OTP, 312 (80\%) had recovered on discharge, 11 (2.8\%) died, and $67(17.2 \%)$ had defaulted. Compared to the baseline data and the recommended minimum standard values, case fatality rate for this study was $2.8 \%$ vs. $7.3 \%$ vs. $>10 \%$ respectively, and the recovery rate was $80 \%$ vs. $73 \%$ vs. $>75 \%$ respectively. Barriers to effective sustainability of the programme included dependence on donor-funds, consistent stock outs of ready-to-use therapeutic food and other supplies, high volunteer dropout, and inadequate monitoring and feedback on defaulters.

Conclusion: OTP improved health outcomes of SAM children in the Eastern Zambia when compared with accepted minimum standards and previous data. Dependent on donor funding and the resultant regular stock-out of supplies can, however, hamper sustainability in the long-term.

Keywords: Severe acute malnutrition, Outpatient Therapeutic Programme, Children, Evaluation, Eastern Zambia, Health outcomes, Case fatality

*Correspondence: kufreokop@gmail.com

School of Public Health, University of the Western Cape, Bellville 7535, South

Africa 


\section{Background}

Nearly 20 million children under five years of age are affected by severe acute malnutrition (SAM) [1]. Malnutrition is a major public health problem, which contributes to high morbidity and mortality rates in Zambia. In 2006, the under-five mortality was at 119 per 1,000 live births, $45 \%$ of children under the age of 5 years were stunted, $5 \%$ wasted and $15 \%$ were underweight [2]. The proportion of children with stunting, wasting and underweight proportions respectively remained unchanged between 2007 (45\%, $5 \%$, and $15 \%)$ and 2014 (40\%, $6 \%$, and $15 \%$ ) based on the recent Zambia Demographic and Health Survey 2013-2014 findings [3].

In 2007, the prevalence of malnutrition in children under 5 years of age in the Eastern Province was at $49.5 \%$ stunting, $3.6 \%$ wasting $12.7 \%$ underweight, and $1.3 \%$ SAM [2]. Although agriculture is a major livelihood in this Province, drought has negatively affected agriculture production thus contributing to food insecurity and disease outbreak [4].

Until recently, hospital-based management option has been the only option available for treating SAM in Zambia and in other developing countries [5]. Due to the cost of hospital admissions, and poor access to clinics, caregivers often avoided hospital admissions [6] for their children. Mothers who access hospital for treatment often brought their malnourished children to the hospital very late, most times with complications [6, 7]. These challenges lead to poor case management resulting in the high case fatality rates recorded in the clinics $[3,6]$. One of the measures taken by the Zambian Government to reduce mortality due to severe malnutrition was to implement the Outpatient Therapeutic Programme (OTP). OTP is considered to be an innovation for treating SAM in children 6 to 59 months of age, with no medical complications as outpatients within their communities, using ready-touse therapeutic food (RUTF) and child care treatment [8-10]. The OTP intervention in Eastern Zambia was first pilot tested as a community-based therapeutic care (CTC) [7] in February 2008, and a full implementation begun in August 2008 [11].

Based on OTP protocol, community volunteers identify children with SAM using the mid-upper-arm circumference (MUAC) tape and the screening for bilateral pitting oedema [11, 12]. Children found with MUAC $<11.5 \mathrm{~cm}$ or with bilateral pitting oedema are referred to the OTP for further screening. Children found with SAM including medical complications are referred to the inpatient care. In the OTP, children receive daily ratio of RUTF, antibiotics, Vitamin A, Folic acid, Anthelminthic and Antimalarial drugs $[1,12]$. The OTP programme has been implemented in other poor resourced countries such as Niger, North and South Sudan, Malawi, Chad and Ethiopia $[5,8,10,12,13]$.
The aim of the OTP intervention was to reduce case fatality rates due to SAM in children in the remote communities in Eastern Zambia. Information on outcomes of OTP implementation at the national and district levels is scarce. In August 2008, a mid-term evaluation of CTC was conducted in the three Eastern Zambia districts (viz. Chipata, Katete, and Patauke) by an external organization $[7,11]$. The purpose of this present study was to evaluate the improvement in health outcomes and fatality rate among children aged 6-59 months diagnosed with SAM in the three districts of Eastern Province, Zambia - post mid-term evaluation. This study addressed the following specific objectives: i) Describes the key indicators for assessing health outcomes of SAM children served in OTP centres between 2008 and 2010 in the districts of Eastern Zambia; and ii) discuss the stakeholders' perspectives and concerns on OTP implementation and sustainability. Findings from this study is believed to describe the current situation of OTP interventions in Eastern Zambia [11].

\section{Methods \\ Study design}

This was a mixed-methods study conducted in two phases. Phase I involved retrospective cohort review of records of children with SAM admitted in the OTP rural health clinics post mid-term evaluation. At Phase II, semistructured key informant interviews were conducted with relevant OTP stakeholders. The key-informant interviews documented the stakeholders' views regarding issues that enhance or hamper effective implementation of sustainable OTP interventions in the districts. To facilitate a periodic evaluation, programme outcome indicators (such as recovery, defaulter and case fatality rates, and weight gain) were compared with baseline data and the recommended minimum standard values.

\section{Study setting and population}

This study was conducted in three districts of the Eastern Province of Zambia, namely, Chipata, Katete and Petauke where OTP has been implemented since 2008. One OTP centre is located in each of the three districts. The estimated populations of these districts in 2007 were 458,700, 233,582 and 315,812 respectively for Chipata, Katete and Petauke $[3,11]$. About $20 \%$ of populations in these districts is estimated to be children, with about one-third of the children in Zambia was reported with wasting in 2008 [2]. Nearly 600 children aged less than five years identified with SAM were admitted for care under OTP implemented in the Rural Health Centres (RHCs) in the districts since its inception [11]. Stakeholders involved in OTP implementation include health workers in RHCs, community leaders, community volunteers, and district health officers and administrators. 
Community volunteers render preventive and counselling services, and also mobilise communities for outreach services.

\section{Sampling and sample size}

The RHCs which implementing outpatients therapeutic programme interventions in the three districts of Eastern Province of Zambia were included in the study. The sample size for the quantitative part of the study was calculated based on the total number of children (i.e. 600 ) served in the three OTP centres. A minimum sample size of 390 was obtained based on the required $3 \%$ absolute precision factor, and a $5 \%$ error of risk. The 390 children aged 6-59 months admitted in these OTP centres between September 1, 2008 and August 30, 2010 were randomly selected from the RHCs, and their records were reviewed.

In the qualitative part of study, all the stakeholders involved in the implementation and administration of OTP services at the community- and the district-levels were listed. Key personnel and community members were selected from the list for the interviews. The stakeholders interviewed included medical officers, health planners, and nutritionist, nurses and community volunteers (Table 1). Steps were taken to ensure that all groups of key stakeholders were represented, and with consideration for gender representation.

Table 1 List of stakeholders interviewed

\begin{tabular}{lllll}
\hline & Chipata & Katete & Petauke & Overall \\
\hline Staff at the OTP Centre & & & & \\
Community Development Officer & 1 & 1 & 1 & 3 \\
Community Health Worker & 1 & 0 & 0 & 1 \\
Community Volunteer & 4 & 4 & 2 & 10 \\
Nurse & 2 & 2 & 2 & 6 \\
Staff total & 8 & 7 & 5 & 20 \\
District/Community Stakeholders & & & & \\
Assistant Community & 0 & 0 & 1 & 1 \\
Development Officer & & & & \\
Community Development Officer & 1 & 3 & 1 & 5 \\
Agriculture Nutrition Officer & 1 & 0 & 1 & 2 \\
Senior Community Development & 1 & 0 & 0 & 1 \\
Officer & & & & \\
Stakeholders total & 3 & 3 & 3 & 9 \\
District Health Staff & & & & \\
District Medical Officer & 1 & 1 & 1 & 1 \\
District Nutritionist & 1 & 1 & 1 & 1 \\
District Health Planner & 1 & 1 & 1 & 1 \\
District Health Staff Total & 3 & 3 & 3 & 3 \\
Total & 14 & 13 & 11 & 38 \\
\hline
\end{tabular}

\section{Data collection}

Summary data collection forms were developed, and pretested in two RHCs, and used to capture patients' records from OTP registers for this study during Phase 1. The data collected were checked for completeness and consistency. In addition, 38 key stakeholders involved in OTP implementation at community and district levels were purposively selected during Phase 2 of the study, and interviewed using validated semi-structured questionnaires. The list of the stakeholders interviewed is presented in Table 1 . The semi-structured questionnaire was first pilot-tested, and administered to respondents in English, since they could speak and write in English. Designated nutritionist and community volunteers in each district attached to the respective OTP centre were trained to facilitate data collection. Data was collected in June 2012.

\section{Inclusion criteria for the study}

Children aged 6-59 months with either marasmus or kwashiorkor admitted in the OTP clinic between September 1, 2008 and August 30, 2010 were randomly selected for the study. Marasmus was described as mid-upper-arm circumference (MUAC) $<11.5 \mathrm{~cm}$, and kwashiorkor was taken as the presence of bilateral pitting oedema. The children with incomplete records initially sampled were excluded, and replaced with those with complete records. Only stakeholders who were health workers, OTP staff or community volunteers and involved in OTP activities in the selected districts for at least period of three months were considered for the study.

\section{OTP outcome indicators evaluated}

The three main outcome indicators evaluated were: i) recovery rate, ii) defaulter rate, and iii) case fatality rate. In addition, average weight gain in $\mathrm{g} / \mathrm{kg} / \mathrm{day}$, and the length of stay (in days) before discharge were also determined.

\section{Operational definitions of OTP Outcome Indicators}

Recovery rate was defined as the proportion the patients discharged as recovered from SAM during a specified period. This was calculated as the number of patients recovered and discharged, divided by the total number of children enrolled in the OTP over the same period multiplied by 100 .

Defaulter rate equals the number of defaulters during a specified period divided by the total number of children enrolled in OTP over the same period multiplied by 100 . Every child admitted into the programme is expected visit OTP every week (with the support of guidance/parent) to receive RUTF, and any medication required according to the programme protocol until recover. If a child misses out on three consecutive weeks 
then they are considered to have defaulted from the programme, and makes the defaulter's list.

Case fatality rate was defined as the number of deaths from SAM during the period under review divided by the number of children admitted with SAM in the same period multiplied by 100 . Another outcome indicator considered was the average weight gain.

Average weight gain ( $/ \mathrm{kg} /$ day) is equal to the difference in weight (in grams) at discharge-and weight at admission (in grams) divide by the product of admission weight $(\mathrm{kg})$ and number of days between admission and discharge. The length of days to recovery was defined as the number of days from admission to recovery or discharge.

To evaluate the OTP intervention, the outcome indicators for this study were compared with the recommended minimum standard indicators (MSI) [14] and the midterm evaluation outcomes (i.e. baseline data) [7]. The minimum SPHERE standard indicators (MSI) are usually used by humanitarian organisations to assess the performance of therapeutic feeding interventions [14].

\section{Stakeholders' perspective on OTP intervention implementation and sustainability}

The stakeholders' views on the implementation and sustainability of OTP were elicited to support programme replanning and decision-making. The information collected included the OTP staff knowledge of service provision according to standard, monitoring and supervision. In addition, data on sustainability challenges or barriers such as financial support/logistics including availability of RUTF and other supplies, referrals, linkages to further care, active participation of community members, community mobilization and outreaches were also obtained.

\section{Data management and analyses \\ Phase I}

The quantitative data was captured in MS Excel worksheet, and imported into and analysed using SPSS version 22. The recommended health outcome indicators were calculated. Descriptive analyses were undertaken and results tabulated based on the districts and individual OTP centre. Data were presented using proportions, and the differences were determined by chi-square test. A $p$-value of $<0.05$ was taken as statistical significance. Comparisons of health outcomes with MSI and available baseline (mid-term evaluation) data were also made.

\section{Phase II}

The data obtained from the semi-structured interviews were transcribed and coded based on three preset themes, namely, implementation, monitoring and sustainability of OTP interventions. The consolidated criteria for reporting qualitative research (COREQ) guidelines were adhered to during this study [15]. The transcripts were first handcoded and the categories and sub-themes were determined. Sub-themes were developed from each of the themes. These sub-themes include knowledge and practice on OTP service provision; sustainability issues-funding support, human resources, community mobilisation, handling defaulters, referrals and linkages; coordination, and monitoring and evaluation. The data were analysed using thematic approach. The development of the themes and sub-themes, and the interpretation of the findings were undertaken by two of the authors.

\section{Results}

\section{Description of the children admitted into OTP}

Table 2 describes the characteristics of the children admitted and discharged in the OTP centres during the two-year period under consideration. Of the 390 children admitted into OTP, 224 (57.4 \%) were females. Majority $(83.9 \%)$ of the children admitted with SAM were aged between 12 and 59 months, and only $16.2 \%$ were aged below 12 months. Overall, about half of the children who were admitted with either kwashiorkor or marasmus were aged 12-23 months. Katete and Chipata

Table 2 Characteristics of children enrolled in OTP centres, the length of stay and number cured

\begin{tabular}{|c|c|c|c|c|}
\hline Variables & Total & Kwashiorkor & Marasmus & $p$-value \\
\hline SAM & $390(100)$ & $238(61.0)$ & $152(39.0)$ & \\
\hline \multicolumn{5}{|l|}{ Gender } \\
\hline Male & $166(42.6)$ & $103(43.3)$ & $63(41.4)$ & \multirow[t]{2}{*}{0.401} \\
\hline Female & $224(57.4)$ & $135(56.7)$ & 89 (58.6) & \\
\hline \multicolumn{5}{|l|}{ Age (in months) } \\
\hline o $6-11$ & $63(16.2)$ & $20(8.4)$ & $43(28.3)$ & \multirow[t]{3}{*}{0.001} \\
\hline o $12-23$ & $193(49.5)$ & $116(48.7)$ & $77(50.7)$ & \\
\hline o 24-59 & $134(34.4)$ & $102(42.5)$ & $32(20.9)$ & \\
\hline \multicolumn{5}{|l|}{ District } \\
\hline o Chipata & $131(33.6)$ & $63(26.5)$ & $68(44.7)$ & \multirow[t]{3}{*}{0.001} \\
\hline o Katete & $129(33.1)$ & $97(40.8)$ & $32(21.1)$ & \\
\hline o Petauke & $130(33.3)$ & $78(32.8)$ & $52(34.2)$ & \\
\hline \multicolumn{5}{|c|}{ Duration of stay in OTP centre } \\
\hline $0<30$ days & $137(35.1)$ & $101(42.4)$ & $36(23.7)$ & \multirow[t]{3}{*}{0.001} \\
\hline o 30-60 days & $106(27.2)$ & $49(20.6)$ & $57(37.5)$ & \\
\hline $0>60$ days & $147(37.7)$ & $88(37.0)$ & $59(38.8)$ & \\
\hline $\begin{array}{l}\text { Mean length of stay } \\
\text { (in days) }\end{array}$ & 44 & 43 & 46 & \\
\hline \multicolumn{5}{|c|}{ Proportion Discharged Cured ${ }^{a}$} \\
\hline All & $312(80.0)$ & $189(79.4)$ & $123(80.9)$ & 0.410 \\
\hline Male & $142(39.4)$ & 86 (36.1) & $56(36.8)$ & 0.492 \\
\hline Female & $170(43.6)$ & $103(43.3)$ & $67(44.1)$ & 0.234 \\
\hline
\end{tabular}

aproportions of those discharged cured is based on the total number $(N=390)$ of SAM children admitted (as the denominator) 
districts had highest numbers of children admitted with kwashiorkor (40.8 \%) and marasmus (44.7\%) respectively, followed by Petuake district (32.8 \% vs. $34.2 \%)$. There were significant differences in the number of children admitted with kwashiorkor and marasmus based on gender and age categories ( $p$-values $<0.05)$.

The average length of stay in the OTP to the time of recovery was 44 days (See Table 2). The length of stay of the children in the OTP centres based on their age categories differs significantly, with nearly $35 \%$ spending less than 30 days, whereas $38 \%$ had stayed for over 60 days before recovery. Majority of children (80\%) were discharged cured during the period under review. More female children (43.6\%) compared to the males (39.4\%) were discharged cured.

\section{Length of stay before recovery and discharge}

The actual length of stay (in days) in the OTP centres before recovery and discharge by district is presented in Fig. 1. Children admitted with marasmus had spent longer days to recovery or exit compared to those admitted with kwashiorkor (50 days vs. 35 days), and this was the pattern in all the districts. More children in Chipata and Katete districts recovered at shorter days (in $<60$ days) compared to the children in Petauke district.

\section{Evaluation of OTP outcomes}

The OTP outcome indicators for this study are presented in Table 3 and described in this section.

\section{Recovery and defaulter Rates}

The recovery and defaulter rates are shown in Table 3. Of the 390 children sampled, 312 (80 \%) recovered (95\% CI range 76-84\%), and 67 (17\%) children (95\% CI range of 14-21\%) had defaulted from OTP intervention. There was no marked difference among the children

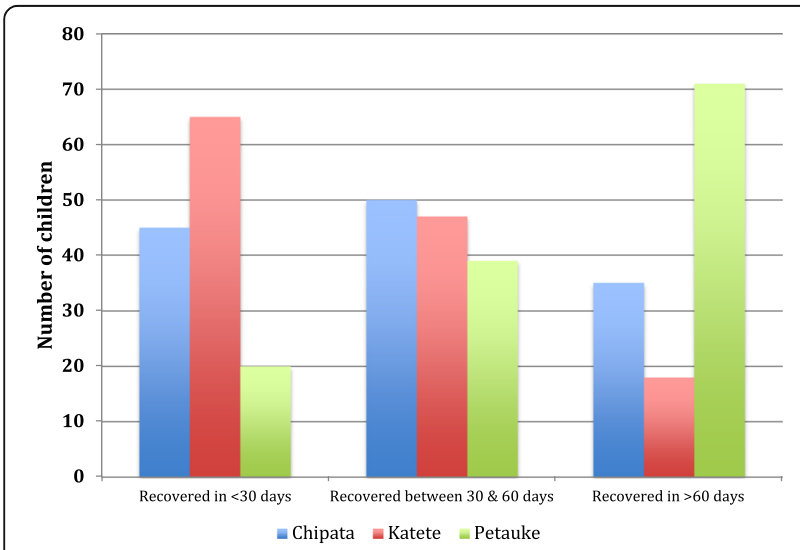

Fig. 1 Length of stay to recovery in the Outpatient Therapeutic Programme by District
Table 3 OTP Outcome indicators - comparison with mid-term evaluation ${ }^{\mathrm{a}}$

\begin{tabular}{lllll}
\hline & Total & $\begin{array}{l}\text { Recovered } \\
\text { (Discharged } \\
\text { cured) }\end{array}$ & Defaulted & $\begin{array}{l}\text { Death/Case } \\
\text { fatality }^{b^{6}}\end{array}$ \\
& $N$ & $n(\%)$ & $n(\%)$ & $n(\%)$ \\
\hline Present study (Overall) & 390 & $312(80.0)$ & $67(17.2)$ & $11(2.8)$ \\
Marasmus & 152 & $114(75.0)$ & $25(16.4)$ & $3(2.0)$ \\
Kwashiorkor & 238 & $181(76.1)$ & $42(17.6)$ & $8(3.4)$ \\
Mid-term Evaluation in & - & $73.2 \%$ & $18.0 \%$ & $7.3 \%$ \\
2008 (Overall), & & & & \\
\hline
\end{tabular}

${ }^{a}$ Comparison is made with mid-term evaluation conducted in 2008

Proportions are specified by row percentages

${ }^{\mathrm{b}}$ For the period considered for evaluation

cBinns and Mutuma, 2008. ${ }^{\mathrm{d}}$ Proportions stated are the combined values for both marasmus and kwashiorkor

with marasmus or kwashiorkor who recovered or defaulted ( $p$-value $>0.05)$.

\section{Case fatality rate}

Eleven children out of 390 died during the period in review, given a case fatality rate of $2.8 \%$. Case fatality rates for individual SAM type were $2.0 \%$ for marasmus, and $3.4 \%$ for kwashiorkor. A comparison of key evaluation findings of this study and that of the baseline evaluation conducted by Valid International (an NGO) [7] in 2008 in the same Eastern Zambia districts is provided in Table 3. Case fatality had decreased from $7.3 \%$ in 2008 (based on mid-term evaluation) to $2.8 \% 2010$ (this study), giving a $4.1 \%$ decrease in this period.

\section{Weight gain}

Sixty one percent of the children in the OTP gained weight within the period. In Table 4, the average weight gain amongst all children admitted with marasmus was 10 g [95 \% CI: 8-17] $/ \mathrm{kg} /$ day while children admitted with kwashiorkor showed no substantial weight gain at discharge-due to loss of oedema. Overall, the average weight gain amongst all admissions with marasmus in Chipata, Katete and Petauke ranged between 9.0 and $10.5 \mathrm{~g} / \mathrm{kg} /$ day. In Katete, $41 \%(98 / 238)$ of children in the programme gained weight, followed by Petauke at $33 \%(78 / 238)$ and Chipata at $26 \%(62 / 238)$.

\section{Improvement in OTP intervention outcomes in the districts}

The programme outcomes were compared with the recommended MSI [14] for Therapeutic centres, and baseline data [7] in order to assess the effectiveness of OTP in the district. Also, programme outcomes were compared in the three OTP centres to ascertain the differences in performance (Table 4). Recovery and mortality rates in this study were all within the recommended standards for therapeutic food centres, whereas 
Table 4 Comparisons of the study outcome indicators with minimum standard indicators (MSI) and the mid-term evaluation data

\begin{tabular}{|c|c|c|c|c|c|c|}
\hline \multicolumn{4}{|l|}{ Programme outcome indicators (SPHERE 2011) [14] } & \multicolumn{3}{|l|}{ Districts } \\
\hline Key indicators for OTP & $\begin{array}{l}\text { Minimum Standard } \\
\text { SPHERE (MSI) }\end{array}$ & $\begin{array}{l}\text { Mid term evaluation } \\
2008^{*}\end{array}$ & Present study & Chipata & Katete & Petauke \\
\hline $\begin{array}{l}\text { Proportion of children from a therapeutic feeding programme } \\
\text { who have died }\end{array}$ & $<10 \%$ & $7 \%$ & $2.8 \%$ & $3.5 \%$ & $0.8 \%$ & $3.8 \%$ \\
\hline $\begin{array}{l}\text { Proportion of exits from a therapeutic feeding programme who } \\
\text { have recovered }\end{array}$ & $>75 \%$ & $73 \%$ & $75.6 \%$ & $76.9 \%$ & $73.8 \%$ & $83.2 \%$ \\
\hline $\begin{array}{l}\text { Proportion of exits from a therapeutic feeding programme who } \\
\text { defaulted }\end{array}$ & $<15 \%$ & $18 \%$ & $17.2 \%$ & $19.2 \%$ & $25.4 \%$ & $6.9 \%$ \\
\hline Minimum mean rate of weight gain ( $\mathrm{g} / \mathrm{kg} /$ day) & $>8 \mathrm{~g}$ & - & $10.0 \mathrm{~g}$ & 10.0 & $9.0 \mathrm{~g}$ & $10.5 \mathrm{~g}$ \\
\hline Average length of stay (in days) & $<60$ & - & 44 & 43 & 38 & 50 \\
\hline
\end{tabular}

* Figures are based on the Mid-term evaluation reported by Binnis and Mutuma [7]

defaulter rate was higher. However, differences in outcomes by district were seen. For example, case fatality was least in Katete (i.e. $0.8 \%$ ), with accompanying highest defaulters rate $(25.4 \%)$. On the other hand, Petuake district recorded the least defaulter rate, and highest recovery rate $(89.2 \%)$ and fatality rate $(3.8 \%)$.

\section{Barriers in the implementation and sustainability of OTP programme}

The results obtained from the semi-structured interviews with the stakeholders are discussed below based on the three key themes considered-namely, barriers in the OTP implementation, monitoring, and sustainability issues.

Barriers listed by the stakeholders included lack of capacity of OTP staff, defaulter rates, poor referrals and linkages to support services for OTP defaulters and discharged clients, lack of financial support and lack of support for monitoring and follow-up of defaulters. The extent of these barriers and how they influence programme implementation and perhaps, sustainability are discussed in this section.

\section{Knowledge and practices of OTP staff}

About $90 \%$ of key informants interviewed had worked at the OTP Centre for more than six months before the study. Most respondents had substantial knowledge about the processes, protocols and management of cases in OTP. The OTP staff and health workers interviewed had received basic training in the OTP programme implementation and were conversant with the admission and discharge criteria for the programme. The stakeholders generally believed that the personnel involved in OTP implementation had good knowledge and service delivery practices. However, the capacity of OTP staff to coordinate, and support monitoring of the services effectively were considered to be sub-optimal.

\section{Handling defaulters}

About $53 \%$ of the key informants indicated that clients defaulted because of stock out of RUTF and drugs, $32 \%$ reported that the clients defaulted due to long distances from home to the OTP centre. Others (15\%) believed that clients defaulted because the caregivers assumed their children had recovered, even though they have not met criteria for discharge. However, very little was done to reduce default rates. Respondents pointed to the challenges of shortage of staff, and consistent lack of supplies, and lack of support for monitoring and followup of defaulters.

\section{Linkages to further care for discharged OTP clients}

The key informants reported that most patients discharged from the OTP centres were linked to other centres for further care. A senior community health officer gave his views as follows:

\section{"Livelihood Programme Centres were established as part of OTP intervention to carter for the needs of the patients who recovered and were discharged from our centres here. This was meant to maintain the recovered children and avoid a relapse to SAM".}

However, only few government-supported centre were available in the community. Majority (90\%) of OTP Centre staff reported that their discharged clients were referred to other nutrition/health programmes (usually donor-supported) implemented in the communities. One common opinion by many OTP centre staff and the community volunteers was:

\section{"Our clients are often referred to one centre named 'Food Security Pack, which is a government programme that provides cereal, legume and fertilizer to vulnerable families".}

Also, most of the respondents (65\%), had indicated that there were no adequate referrals made to the recommended 'livelihood programme centres', indicating poor linkages between the OTP and livelihood programmes. It was also reported that that the communities were adequately involved in community mobilisation to facilitate early identification, and referral of cases of SAM to health 
facilities. However, monitoring of OTP clients and feedback to the community was not adequately coordinated due to lack of funding.

\section{Monitoring and supervision issues}

The stakeholders reported that the children in OTP care were monitored on a weekly basis to assess improvement (or otherwise) in weight, height, MUAC, and response to therapeutic treatment. The nurses in each of the districts reported in consistently. One of the nurses stated this as follows:

"The children are monitored on a weekly basis. Their weight, height, MUAC, and response to therapeutic treatment are recorded each week".

Although information generated from monitoring OTP activities was reportedly shared during some of the review and staff meetings, findings from the semi-structured interview revealed that inadequate monitoring and feedback among OTP staff and the community. Staff at OTP centres, however, reported that they regularly assess children to identify those that failed to attain desired minimum weight gain. Up to $70 \%$ of the staff reported that such children were either referred for further investigation in the next level of care (District Hospitals), or provided caretakers of affected children with infant feeding counselling and advice. The OTP staff at district indicated that OTP centre staff capacities were built through involvement in monitoring and review meetings. A designated programme coordinator oversees the implementation of outpatient therapeutic programme at all levels (i.e. OTP Centres, district and provincial) with the support of the supervisors. It was also reported that the supervision of community activities were hindered by the lack of a functioning motorbike or support for transportation.

\section{Financial support, logistics and community mobilisation}

One key issue on the implementation and sustainability of OTP mentioned by the stakeholders was the challenge of short-term donor-dependent funding or financial support. This was believed to affect the effective programme implementation and monitoring. Other issues mentioned which were believed to hinder sustainability were lack of re-training for OTP staff, inadequate monitoring and review meetings. Although budget support for monitoring and supervision were included in the annual action plan for the district, long term support of OTP activities were not fully considered.

Issues of stock out of supplies and dropout of community volunteers were sustainability challenges raised by the stakeholders. The OTP Centre staff and the District health office staff reported prolong stock outs of OTP supplies such as MUAC tapes, RUTF and drugs. About $50 \%$ of key informants indicated that lack of motivation or incentives was the reason for volunteer dropout, $45 \%$ indicated that too much work in OTP. In some of the communities, lack of means of mobility for volunteers hampered volunteers' activity. Under this programme, volunteers were to be given bicycles to support their work [7]. At the time of the study, not all volunteers were in possession of a bicycle. Volunteers also had complained of lack of maintenance of the bicycles they were provided, making transportation challenging.

\section{Discussion}

The findings of this study indicate an improvement in health outcomes and reduction in mortality (or case fatality) rate in children admitted in OTP centres when compared to the minimum standards and baseline data. Four out of the five indicators considered in this evaluation, namely recovery rate $(80 \%)$, death rate $(2.8 \%)$, average weight gain $(10 \mathrm{~g} / \mathrm{kg} /$ day $)$, and average length of stay to recover (44 days) exceeded the MSI for OTP [7]. The defaulter rate $(17 \%)$ was however higher than that of the minimum standard expected. This was attributed to stock outs of RTUF, poor community mobilization and caregiver's attitudes.

Furthermore, case fatality rate specifically have decreased from $7.3 \%$ in 2008 to $2.8 \% 2010$ based on comparison between the mid-term evaluation and this study findings, giving a $4.1 \%$ decrease in child mortality rate in these three centres. These comparisons give an indication of a substantial improvement in health outcomes and reduction in case fatality between 2008 and 2010. Further comparison of the overall programme outcomes with the MSI in the three OTP centres for period of the evaluation points to a remarkable improvement in the health outcomes.

Findings from this study were similar to a study conducted in Ethiopia that reported $85 \%, 4 \%$ and $5 \%$ recovery, mortality and defaulter rates respectively [5]. However, the death rate in the present study is lower, whereas the defaulter rate is higher than the aforementioned Ethiopian study. The variation in the findings between these two studies may be due to the study designs. The current study, had larger sample size of 390 children recruited over a period of 2 years, compared to the 170 children recruited within a period of five months. The larger sample size coupled with a longer duration of study (2 years) might have resulted in data inaccuracies.

The high recovery rate, and low death rate has an implication for effective follow up if these outcomes must be sustained. Sustaining the improved outcomes is unlikely, as there was a weak system of linkages to the existing likelihood programmes, and poor follow up on defaulters. The low mortality rate of $2.8 \%$ can be attributed to improved case management and timely detection of cases [16]. The high defaulter rate is linked 
to the challenge of accessibility and acceptability of intervention in the community. Caregivers exiting OTP centres with the assumption that their children have recovered added to the number of defaulters. This points to a critical need for health education at community level and at OTP centres for OTP caregivers [12].

The high defaulter rate could also result in child mortality as untraced defaulters may likely die at home without being recorded. To ameliorate the dangers of defaulters, and relapse of those cured or discharge, OTP intervention should undertake periodic community-level monitoring to link children discharged to further care and to follow up defaulters to assess their nutritional status at intervals. This is cost intensive though, and therefore should be planned and budgeted for at the onset of the programme implementation. However, this may be difficult to achieve in the immediate since funding for OTP interventions currently are donor-dependent.

Prolonged recovery rates (beyond 60 days) particularly in Petuake and Chipata districts would impact on the cost of OTP delivery since more resources are required to maintain the patients. On the other hand, longer stay of clients in OTP indicates poor monitoring and inadequate adherence to protocols at health facility. A study conducted by Collins and colleagues reported a reduction in average length of stay when rehabilitation was done at home [16], pointing to the need to encourage care at home, and monitoring at facility, earlier advocated [8].

\section{Implications for programme and policy}

In order for nutrition programmes to be sustainable, internal and reliable funding is required [16]. Dependence on donor funding for community-based nutrition programmes cannot be sustainable. To ensure sustainability of nutrition programmes such as OTP, it is therefore essential for the Zambian Government to ensure adequate budgetary allocation to the Ministry of Health to support the funding of OTP interventions at the districts.

Furthermore, in a scarce resource environment like Zambia communities, efforts should be made to encourage community-based efforts for programme management to reduce cost and increase sustainability. In this regards, effective and sustainable nutrition intervention can be achieved through community-directed approach and advocacy for ownership. Adequate community participation beginning from programme conception, planning and implementation can facilitate effective community resource mobilisation and community buy-in. In addition, OTP intervention should be integrated and linked to curative, community-directed prevention and child survival programmes to maximize coverage and resource leverage [8].

The increasing dropouts of volunteers reported in the study can be a challenge for sustainability of OTP activities, and could lead to high defaulter rates. Incentives for volunteers and financial support in Katete had enhanced retention of volunteers in the past [7]. In Katete district, volunteers were given financial support as incentive for transportation. This incentive commenced in February 2008, were offered through the district nutrition fund supported by an NGO, and there were no drop out among the volunteers. There is need to provide support in the form of bicycles, transport stipend, souvenirs, or community recognitions as incentives for volunteers. This will not only motivate volunteers living in remote and distant communities, but also will boost their commitment and reduce attrition. However, it is important to develop strategies to engage with the community for ownership of OTP activities in other to reduce volunteer dropout and defaulters rate.

Adequate information sharing and learning during interventions seems to be lacking in nutrition programmes. For instance, although OTP activities were monitored, information sharing between OTP staff at the national, districts and community levels for lessons learnt were inadequate. Sharing lessons learnt and the best practices among staff involved in the OTP implementation can improve service delivery and management of defaulters. Although the staff and community volunteer were trained for community mobilization and CTC service delivery, this may not be adequate for effectiveness and efficiency. A more didactic training on communitybased outpatient nutrition intervention programming is therefore required. This should be incorporated into the existing training curricula for lower and middle cadre health care professionals [16].

\section{Strength and limitations of the study}

In addition to review of clinic records and semi-structured interviews with staff, the study involved all key stakeholders and beneficiaries of OTP intervention to objectively assess the outcome of the programme. Limitations of the study include the use of different volunteers to collect measurements such as MUAC and weight from the study participants each time when measurements are collected. Although they had received initial training, the tendency to introduce errors in the measurements is high. In addition, the study was conducted in only one health facility in each of the three districts where OTP is implemented, and may not be a representation of the situation in the entire districts of Eastern Zambia. However, the findings from this study provided useful information that can be used to improve the implementation of the OTP programme.

\section{Conclusion and recommendations}

OTP intervention has improved the health outcomes of children admitted with SAM in the Eastern districts of 
Zambia, when compared with accepted minimum standards and previous data. Increasing dependence on donor funding for OTP, the constraints of regular stock-out of supplies, and lack of monitoring and follow-up of defaulters can hamper sustainability in the long-term.

\section{Abbreviations}

CTC: Community-based therapeutic care; MIS: Minimum standard indicators; MUAC: Mid-upper-arm circumference; OTP: Therapeutic Programme; RHCs: Rural Health Centres; RUTF: Ready-to-use therapeutic food; SAM: Severe acute malnutrition; ZMOH: Ministry of Health

\section{Acknowledgements}

The authors would like to acknowledge Ms. Mary Kaoma of Zambia Integrated of Systems Strengthening Programme for logistic support during data collection. We thank also the Zambian Eastern district and provincial nutritionists that participated in data collection in the three districts Our appreciation goes to the Ministry of Health offices in the headquarters, provincial and district for approval to work with their staff at the facilities, and to all the study participants.

\section{Funding}

There was no official funding support for this study.

\section{Availability of data and materials}

All the data are contained within the manuscript and are available from the corresponding author upon reasonable request.

\section{Authors' contributions}

NM conceptualized, designed and implemented the study. He also involved in data analyses and the development of the draft manuscript. OKJ was involved in data analyses, interpretation and writing of the manuscript. PT was involved in the design and supervision of the study, data analyses and reviewing of the manuscript drafts. All authors read and approved the final manuscript.

\section{Authors' information}

Mike Nwanza, MPH: School of Public Health, University of the Western Cape, South Africa; and National Food and Nutrition Commission, Lusaka, Zambia (email address: mikemwanza@gmail.com) Kufre Joseph Okop, MPH: Doctoral Candidate, School of Public Health, University of the Western Cape, South Africa; and doctoral research fellow with the Chronic Disease Initiative for Africa (CDIA), University of Cape Town, South Africa. Email: kufreokop@gmail.com. Thandi Puoane, MPH, DrPH: Professor Emeritus, School of Public Health, University of the Western Cape, South Africa. Email: tpuoane@uwc.ac.za.

\section{Competing interests}

The authors declare that they have no competing interests.

\section{Consent for publication}

There was no individual person's data collected during the study that could have warranted consent of publication. Participants provided consent for their quotes to be published.

\section{Ethics approval and consent to participate}

Permission to conduct the study was obtained from the University of Zambia Ethics Committee and Ministry of Health of Zambia, and the University of the Western Cape Ethics committee. In phase I, the Provincial Medical Officer and the respective District Medical Officers also gave permission to review the records of the children in their respective OTP Centres. The children's records were provided with numbers for identification instead of names, and none of the children were interviewed in person. In phase II, all participants that were interviewed provided written informed consent by signing a consent form, after accepting verbally to participate in the study. Participants were duly informed that participation in the study was voluntary. Information obtained during the study was kept confidential.

\section{References}

1. WHO. Community-based management of severe acute malnutrition. A Joint Statement by the World Health Organization, the World Food Programme, the United Nations System Standing Committee on Nutrition and the United Nations Children's Fund. 2007. http://www.who.int/nutrition/ topics/Statement_community_based_man_sev_acute_mal_eng.pdf. Accessed 11 July 2016

2. Central Statistical Office (CSO), Ministry of Health (MOH) TDRC (TDRC), University of Zambia: Zambia Demographic and Health Survey 2007. Calverton, Maryland, USA; 2009

3. Central Statistical Office (CSO) [Zambia], Ministry of Health ( $\mathrm{MOH})$ [Zambia], ICF International: Zambia Demographic and Health Survey 2013-14. https://www.dhsprogram.com/pubs/pdf/FR304/FR304.pdf. Accessed 12 July 2016.

4. Zambia Demographic and Health Survey, 2001-2002 [https://www.datafirst. uct.ac.za/dataportal/index.php/catalog/378]. Accessed 12 July 2016.

5. Collins S, Sadler K. Outpatient care for severely malnourished children in emergency relief programmes: A retrospective cohort study. Lancet. 2002;360:1824-30

6. Khunga H, Okop KJ, Puoane T. Perceptions of mothers and caregivers regarding the detection and treatment of severely malnourished children in kanchele, kalomo district, zambia. Africa J Nurs Midwifery. 2014;16:98-111.

7. Binnis Paul and Mutuma Sandra: Community-Based Therapeutic Care Mid Term Evaluation Chipata, Katete \& Petauke Districts Eastern Province Zambia. Valid International, Luzaka 2008; p-1-19.

8. Collins S, Dent N, Binns P, Bahwere P, Sadler K, Hallam A. Management of severe acute malnutrition in children. Lancet. 2006;368:1992-2000.

9. Hendricks KM. Ready-to-use therapeutic food for prevention of childhood undernutrition. Nutr Rev. 2010;68:429-35.

10. Chaiken MS, Deconinck H, Degefie T. The promise of a community-based approach to managing severe malnutrition: A case study from Ethiopia. Food Nutr Bull. 2006;27:95-104.

11. Mike M: Mwanza Mike. Evaluation of the outpatient therapeutic programme for management of severe acute malnutrition in three districts of eastern province, Zambia. Thesis. University of the Western Cape, South Africa; 2013. http://etd.uwc.ac.za/xmlui/handle/11394/3919. Accessed 10 Sept 2016.

12. Valerie Gatchell VF and Pual-Ress T: The Sustainability of Community-Based Therapeutic Care (OTP) in Non- Acute Emergency Contexts: Technical Background Paper. 2005. Dublin, Ireland

13. Globler-Tanner C. \& Collins S: Community Therapeutic Care (CTC): A New Approach to Managing Acute Malnutrition in Emergencies and Beyond. Washington DC. 2004. http://www.popline.org/node/237697. Accessed 13 July 2016.

14. The Sphere Project: Humanitarian Charter and Minimum Standards in Humanitarian Response. Volume 1; 2011. DOI 10.3362/9781908176202.

15. Tong A, Sainsbury P, Craig J. Consolidated criteria for reporting qualitative research (COREQ): A 32-item checklist for interviews and focus groups. Int J Qual Heal Care. 2007:19:349-57.

16. Collins S, Sadler K, Dent N, Khara T, Guerrero S, Myatt M, Saboya M, Walsh A Key issues in the success of community-based management of severe malnutrition. Food Nutr Bull. 2006:27:S49-82.

\section{Submit your next manuscript to BioMed Central} and we will help you at every step:

- We accept pre-submission inquiries

- Our selector tool helps you to find the most relevant journal

- We provide round the clock customer support

- Convenient online submission

- Thorough peer review

- Inclusion in PubMed and all major indexing services

- Maximum visibility for your research

Submit your manuscript at www.biomedcentral.com/submit
Biomed Central 